\title{
Modeling of Shot Noise in Resonant Tunneling Structures
}

\author{
G. IANNACCONE *, M. MACUCCI and B. PELLEGRINI
}

Dipartimento di Ingegneria dell' Informazione: Università degli studi di Pisa, Via Diotisalvi 2, I-56126 Pisa, Italy

\begin{abstract}
In this paper, we present insights into the transport properties and the geometrical structure of resonant tunneling devices that can be obtained by the study of their noise properties. We stress the importance of including noise behavior among the objectives of device simulations. The reason is twofold: on one hand, as the number of carriers involved in device operation decreases, fluctuations become more relevant; on the other hand, in devices whose functionality is based on quantum effects, noise properties strongly depend on the details of device geometry.
\end{abstract}

Keywords: Resonant tunneling, nanoelectronics, device modeling, noise modeling, shot noise

\section{INTRODUCTION}

In recent years, noise characterization has emerged as a powerful tool for obtaining information about the structure and the transport properties of nanoscale devices complementary to those given by the DC characteristics and the small signal AC response. In fact, since the number of charge carriers involved in device operation is decreased with respect to semiclassical devices, it is apparent that fluctuations, and in particular those due to the granularity of charge (the so-called "shot noise"), acquire an increasing importance. Furthermore, noise in such structures exhibits a behavior which is strongly dependent upon the details of device geometry.
Therefore, it is important to extend the domain of device simulations to noise properties. Here, we focus on resonant tunneling structures, for which a greater number of experimental data is available.

Since the pioneering work of Lesovik [1] and the first experimental results of $\mathrm{Li}$ and coworkers [2], many theoretical studies [3-6] and experimental results $[5,7,8]$ have appeared in the literature, assessing that the power spectral density $S$ of the noise current in such devices may be suppressed down to half the "full" shot noise value $S_{\text {full }}=$ $2 q\langle i\rangle$, due to correlation in the motion of individual electrons introduced by electrostatic force and/or Pauli exclusion.

In this paper, we presents insights into the transport properties and the geometrical structure

* Corresponding author. 
of resonant tunneling devices that can be obtained by the study of their noise properties. Our calculations are based on a model for transport and noise in generic resonant tunneling structures which has been presented elsewhere $[6,9]$, so that only a brief description will be given in Section 2, while the interested reader can find analytical details in the cited papers. Numerical results and a comparison with available experimental results will be shown in Section 3, while a discussion of the results obtained and of the future developments ends the paper.

\section{MODEL}

Let us consider the one-dimensional structure sketched in Figure 1: it consists of three regions $\Omega_{l}, \Omega_{w}$ and $\Omega_{r}$, i.e., the left reservoir, the well region and the right reservoir, respectively, that are only weakly coupled through the two tunneling barriers 1 and 2. Moreover, we suppose that electron transport is well described in terms of sequential tunneling: an electron in $\Omega_{1}$ traverses barrier 1 , loses phase coherence and relaxes to a quasi-equilibrium energy distribution in the well

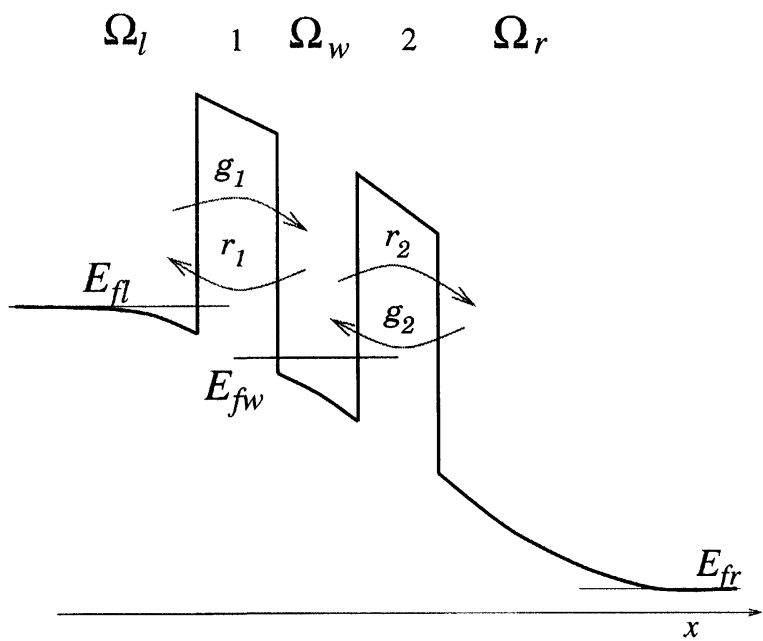

FIGURE 1 A generic resonant tunneling structure consists of three isolated regions $\Omega_{l}, \Omega_{w}, \Omega_{l}$ weakly coupled by tunneling barriers, indicated here with 1 and 2 . Coupling between different regions has to be small enough to be treated with first order perturbation theory. region $\Omega_{w}$, then traverses barrier 2 and leaves through $\Omega_{r}$. Such hypothesis is very reasonable, except at millikelvin temperatures, where inelastic processes are strongly suppressed and no more effective in thermalizing electrons in the well.

The typical resonant current peaks in the $I-V$ curve are due to the shape of the density of longitudinal states in $\Omega_{w}$, which is strongly affected by confinement: for the material parameters considered here, it has a single narrow peak in correspondence of the allowed longitudinal energy level of $\Omega_{w}$; the rate of inelastic scattering processes affects the width of such peak and in our model this effect is taken into account through a phenomenological parameter, the mean free path $l$, which plays the role of a relaxation length for phase and energy. The density of states in the well is calculated using a compact formula derived in Ref. [11].

A state in $\Omega_{s}(s=l, r, w)$ is characterized by its longitudinal energy $E$, its transverse wave vector $\boldsymbol{k}_{\boldsymbol{T}}$ and its spin $\sigma$. Tunneling is treated as a transition between levels in different regions [10] in which $E, \boldsymbol{k}_{\boldsymbol{T}}$ and $\sigma$ are conserved.

Following Davies et al. [4], we introduce the "generation" rates $g_{1}$ and $g_{2}$, i.e., the transition rates from $\Omega_{l}$ to $\Omega_{w}$ and from $\Omega_{r}$ to $\Omega_{w}$, obtained by summing the transition rates given by the Fermi golden rule over all pairs of occupied states in the initial region and available states in the final region. Analogously, we define the "recombination" rates, i.e., the transition rates $r_{1}$, from $\Omega_{w}$ and $\Omega_{l}$, and $r_{2}$, from $\Omega_{w}$ to $\Omega_{r}$, the total generation rate $g=g_{1}+g_{2}$ and the total recombination rate $r=r_{1}+r_{2}$. We refer the reader to Ref. [6] for detailed analytical expression.

The occupation factor in the well, which, under the assumption of complete relaxation, depends only on the value of the quasi-fermi level $E_{f w}$ in the well, has to be calculated in the steady state condition, i.e., by imposing $\langle g\rangle=\langle r\rangle$, where we denote the steady state value of a generic quantity $a$ as $\langle a\rangle$. The average current is

$$
\langle i\rangle=q\left\langle g_{1}-r_{1}\right\rangle=q\left\langle r_{2}-g_{2}\right\rangle .
$$


The problem of transport is solved self-consistently, since the charge accumulated in the well affects the conduction band profile of the structure. We also need to obtain the complete curve of $g$ and $r$ as a function of the number of electrons in the well $N$ (that is to say, of the quasi-Fermi level in the well). Then, we expand $g$ and $r$ to first order in $N$, around the steady state value $\tilde{N}$ and introduce the following characteristic times:

$$
\begin{gathered}
\frac{1}{\tau_{g 1}} \equiv-\left.\frac{d g_{1}}{d N}\right|_{N=\tilde{N}}, \quad \frac{1}{\tau_{g 2}} \equiv-\left.\frac{d g_{2}}{d N}\right|_{N=\tilde{N}}, \\
\left.\frac{1}{\tau_{r 1}} \equiv \frac{d r_{1}}{d N}\right|_{N=\tilde{N}},\left.\quad \frac{1}{\tau_{r 2}} \equiv \frac{d r_{2}}{d N}\right|_{N=\tilde{N}} ;
\end{gathered}
$$

from which we can define

$$
\tau_{1}^{-1} \equiv \tau_{g 1}^{-1}+\tau_{r 1}^{-1}, \quad \tau_{2}^{-1} \equiv \tau_{g 2}^{-1}+\tau_{r 2}^{-1}
$$

and

$$
\tau^{-1}=\tau_{1}^{-1}+\tau_{2}^{-1}
$$

The power spectral density $S(\omega)$ of the noise current at low frequency $(\omega \tau \ll 1)$ can be written as

$$
S(\omega)=2 q^{2}\left(\frac{\tau^{2}\left\langle g_{1}+r_{1}\right\rangle}{\tau_{2}^{2}}+\frac{\tau^{2}\left\langle g_{2}+r_{2}\right\rangle}{\tau_{1}^{2}}\right) ;
$$

a detailed derivation of this result can be found in Ref. [6]; it suffices here to say that no additional hypothesis is required to arrive at (6).

An important parameter is the noise suppression factor $\gamma$, also called "Fano factor", i.e., the ratio between $S(\omega)$ and the "full shot" noise value $S_{\text {full }}=2 q\langle i\rangle$. From (1), (4), (5) and (6), it is apparent that it can reach a minimum of $\gamma=0.5$ if $\tau_{1}=\tau_{2},\left\langle g_{2}\right\rangle \ll\left\langle r_{2}\right\rangle$ and $\left\langle r_{1}\right\rangle \ll\left\langle g_{1}\right\rangle$.

\section{RESULTS}

We consider a device with the following layer structure: a Si-doped $\left(N_{d}=1.4 \times 10^{18} \mathrm{~cm}^{-3}\right) 500$ nm-thick GaAs buffer layer, an undoped $20 \mathrm{~nm}$ - thick GaAs spacer layer to prevent silicon diffusion into the barrier, an undoped $11.5 \mathrm{~nm}$-thick $\mathrm{AlGaAs}$ barrier (barrier 2), an undoped $5 \mathrm{~nm}$ thick GaAs quantum well, an undoped $10 \mathrm{~nm}$ thick AlGaAs barrier (barrier 1), an undoped 10 nm-thick GaAs spacer layer and a Si-doped 500 nm-thick cap layer. The aluminum mole fraction in both barriers is 0.36 . Noise measurements on such structure as a function of current and temperature have been presented in Ref. [8].

In Figure 2 the experimental forward $I-V$ characteristic at $77 \mathrm{~K}$ is compared with the simulation result. The mean free path is chosen as a fitting parameter, and is equal to $15 \mathrm{~nm}$. We have found that the so called "peak to valley ratio", i.e., the ratio between the peak current and the valley current, is almost linearly dependent on the mean free path, while the other parameters are practically independent. For the structure being investigated, a mean free path close to $15 \mathrm{~nm}$ seems to be the best fit at all considered temperatures (since the mean free path accounts for all randomizing effects, it seems that some temperature independent cause, e.g., interface roughness, is predominant); therefore, the decrease of the peak to valley ratio with increasing temperature seems to be due only to the Fermi distribution spreading.

The noise current power spectral density $S$ as a function of current is plotted in Figure 3 for three different temperatures, and for very low bias. As expected, at equilibrium $S$ tends to the thermal

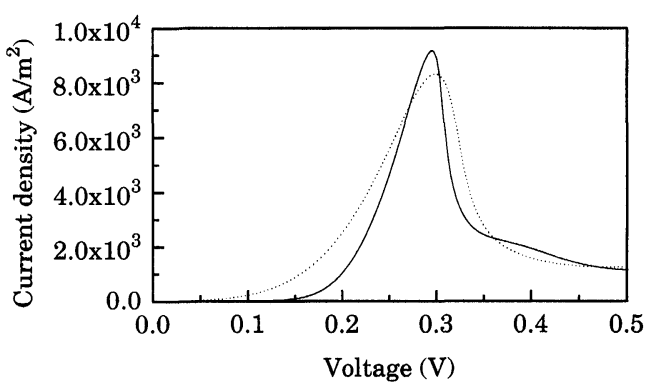

FIGURE 2 Forward $I-V$ curves at $77 \mathrm{~K}$ : comparison between experiment (solid) and simulation with mean free path $15 \mathrm{~nm}$ (dotted). 


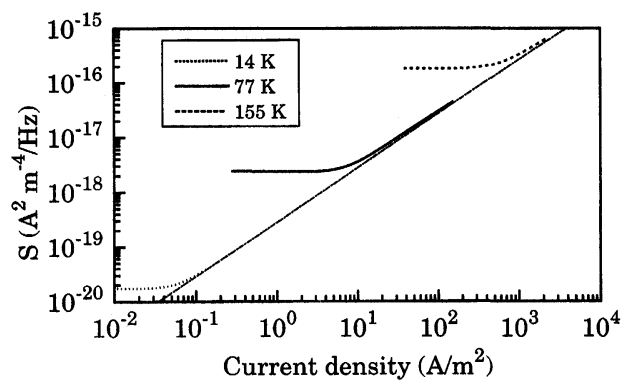

FIGURE 3 Noise current power spectral density as a function of current density for very low biases at three different temperatures: $14 \mathrm{~K}$ (dotted), $77 \mathrm{~K}$ (solid), $155 \mathrm{~K}$ (dashed). The straight line is $S_{\text {full }}=2 q\langle i\rangle$.

noise value $S=4 G k_{B} T$, while, as the bias is increased, it approaches the $2 q\langle i\rangle$ curve. This is an important check for the validity of our noise model.

In Figure 4, the noise suppression factor $\gamma$ as a function of current in the resonant region of the $I-V$ curve is plotted for different temperatures and compared with the experimental results. As
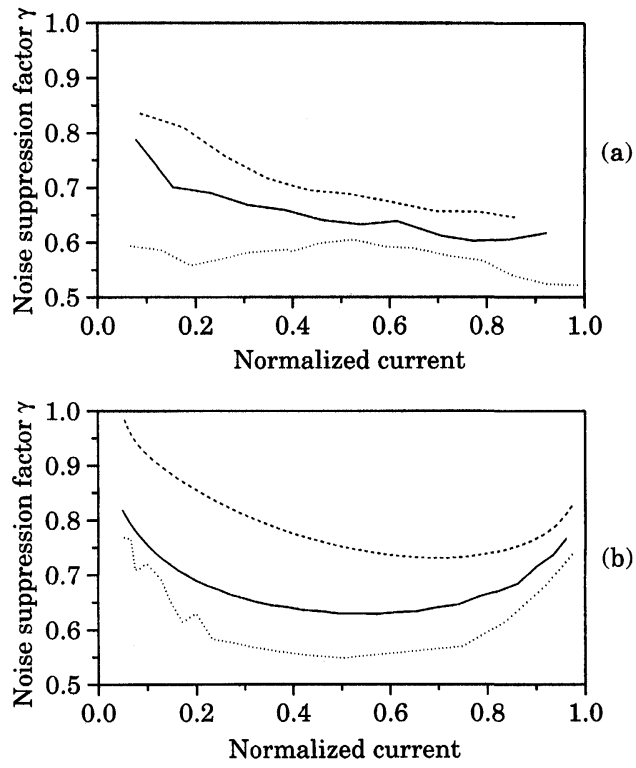

FIGURE 4 Noise suppression factor as a function of normalized current at the temperatures of $14 \mathrm{~K}$ (dotted), $77 \mathrm{~K}$ (solid), $155 \mathrm{~K}$ (dashed): comparison between experiments (a) and simulations (b). Biases lower than that of the resonant peak are considered and the current at the resonant peak is taken as unity. can be seen, $\gamma$ increases with increasing temperature. This behavior is described in [6].

For the same structure, in Figure 5, $\gamma$ is plotted as a function of the applied voltage at the temperature of $77 \mathrm{~K}$. For bias voltages in the negative differential resistance (NDR) region, enhanced shot noise is to be expected, while for voltages greater than that corresponding to the valley current, full shot noise is obtained (Pauli exclusion and Coulomb repulsion are no more effective in correlating current pulses). For the latter result, an experimental evidence can be found in [8], while, for the former, measurements are in progress.

The enhanced shot noise in the NDR region depends on the fact that the characteristic time $\tau_{g 1}$ is negative, i.e., the transition rate $g_{1}$ increase with increasing $N$. The reason is that the peak in the density of states is below the conduction band bottom of the cathode: when an electron enters the well, the conduction band bottom of the well is raised, and more states are available for tunneling from the left electrode, so that electron crossings through the whole structure are positively correlated.

\section{DISCUSSION}

We have shown that numerical modeling of noise in resonant tunneling devices provides new insights into device physics and structure, allows us to recover the results of experiments with a reason-

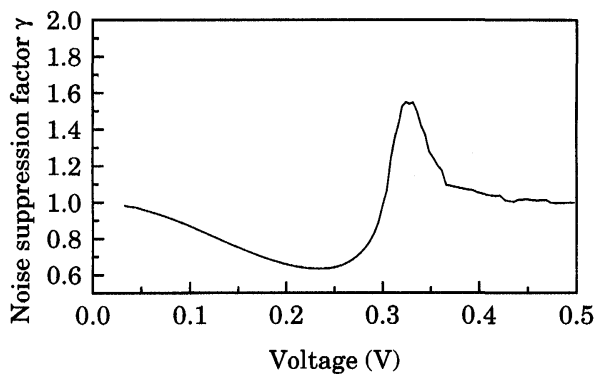

FIGURE 5 Theoretical noise suppression factor at $77 \mathrm{~K}$ as a function of voltage. 
able accuracy and to predict interesting new results. The results obtained give us confidence in the model for transport and noise in resonant tunneling structures described in $[6,9,11]$.

Further developments will include a more refined model to predict experimental data with better accuracy; in particular, greater attention will be devoted to the connection between regions in which transport can be described as semiclassical and regions in which the quantum nature of transport must be taken into full account.

\section{Acknowledgements}

This work has been supported by the Ministry for the University and Scientific and Technological Research of Italy and by the Italian National Research Council (CNR).

\section{References}

[1] Lesovik, G. B. (1989). "Excess Quantum Noise in 2D Ballistic Point Contacts", JETP Lett., 49, 592-594 (Pis'ma Zh. Eksp. Teor. Fiz., 49, 513).

[2] Li, Y. P., Zaslavsky, A., Tsui, D. C., Santos, M. and Shayegan, M. (1990). "Noise Characteristic of DoubleBarrier Resonant-Tunneling Structures Below $10 \mathrm{KHz}$ ", Phys. Rev. B, 41, 8388-8391.

[3] Büttiker, M. (1992). "Scattering Theory of Current and Intensity Noise Correlations in Conductors and Wave Guides", Phys. Rev. B, 46, 12485-12507.

[4] Davies, J. H., Hyldgaard, P., Hershfield, S. and Wilkins, J. W. (1992). "Classical Theory of Shot Noise in Resonant Tunneling", Phys. Rev. B, 46, 9620-9633.

[5] Brown, E. R. (1992). "Analytic Model of Shot Noise in Double-Barrier Resonant Tunneling Diodes", IEEE Trans. Electron Devices, 39, 2686-2693.

[6] Iannaccone, G., Macucci, M. and Pellegrini, B. (1997) "Shot Noise in Resonant Tunneling Structures", Phys. Rev. B, 55, 4539-4550.
[7] Liu, H. C., Li, J., Aers, G. C., Leavens, C. R. and Buchanan, M. (1995). "Shot-Noise Suppression in Resonant Tunneling", Phys. Rev. B, 51, 5116-5120.

[8] Ciambrone, P., Macucci, M., Iannaccone, G., Pellegrini, B., Sorba, L., Lazzarino, M. and Beltram, F. (1995). "Noise Measurements in Resonant Tunneling Structures as a Function of Current and Temperature", Electronics Lett., 31, 503-504.

[9] Iannaccone, G. and Pellegrini, B. (1995). "Unified Model to Electron Transport in Double Barrier Structures", Phys. Rev. B, 52, 17406-17412.

[10] Bardeen, J. (1961). "Tunneling from a Many-Particle Point of View", Phys. Rev. Lett., 6, 57-59.

[11] Iannaccone, G. and Pellegrini, B. (1996). "Compact Formula for the Density of States in a Quantum Well", Phys. Rev. B, 53, 2020-2025.

\section{Authors' Biographies}

G. Iannaccone is serving on the faculty of the Electrical Engineering Department (Dipartimento di Ingegneria dell' Informazione) at the University of Pisa, Italy. His research interests include transport and noise modeling in mesoscopic and heterostructure devices, architectures and devices for nanoscale integrated circuits.

M. Macucci is serving on the faculty of the Electrical Engineering Department (Dipartimento di Ingegneria dell' Informazione) at the University of Pisa, Italy. His research interests include quantum-interference and single-electron devices, Coulomb Blockade phenomena, modeling and measurements of noise in electron devices.

B. Pellegrini is Professor of Electronics at the Electrical Engineering Department (Dipartimento di Ingegneria dell' Informazione) of the University of Pisa, Italy. His recent research interest include modeling and characterization of transport and noise in electron devices. 

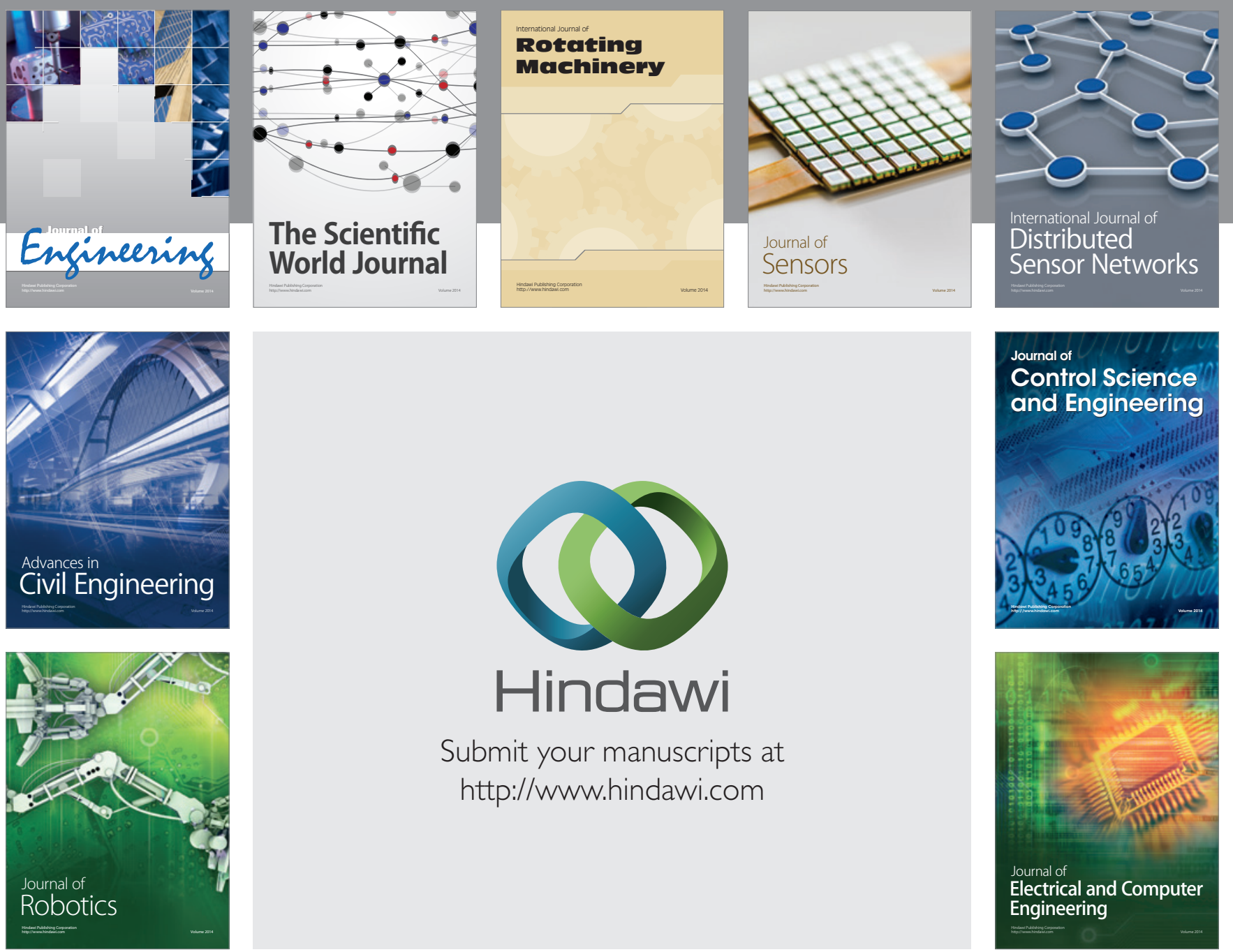

Submit your manuscripts at

http://www.hindawi.com
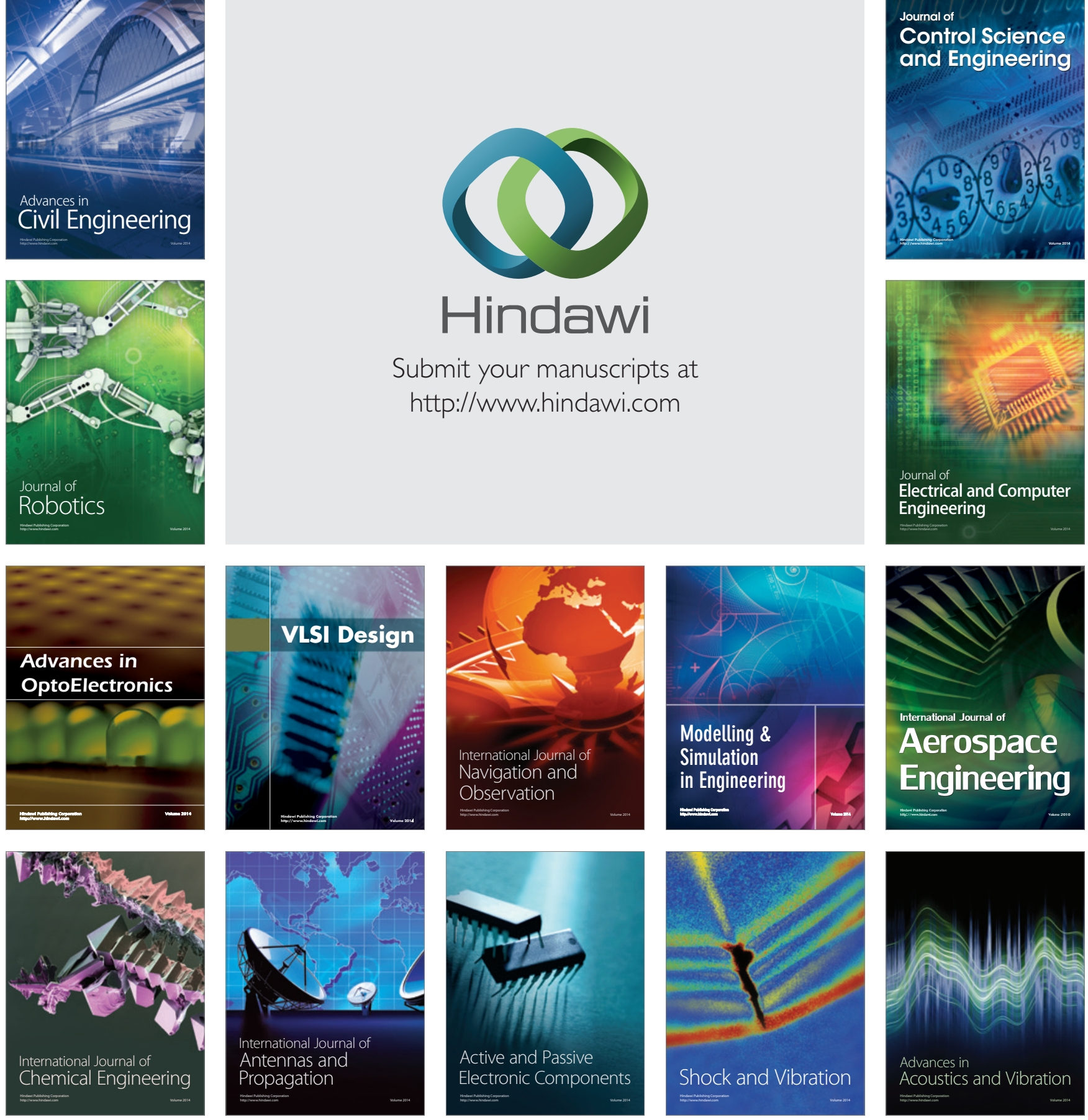